\title{
SISTEMAS DE INOVAÇÃO E O COMPLEXO ECONÔMICO E INDUSTRIAL DA SAÚDE: ASPECTOS TEÓRICOS
}

\author{
Jacilene dos Santos Silva \\ Mestrado em Economia Aplicada pela Universidade Federal de Alagoas. \\ Maceió, AL, Brasil \\ Vanessa Juliana de Mendonça Araújo \\ Mestrado em Economia Aplicada pela Universidade Federal de Alagoas \\ Maceió, AL, Brasil \\ Francisco José Peixoto Rosário \\ Professor da Faculdade de Economia, Administração e Contabilidade (FEAC) \\ Universidade Federal de Alagoas (UFAL), \\ Maceió, AL, Brasil. \\ Maria Cecília Junqueira Lustosa \\ Professora da Faculdade de Economia, Administração e Contabilidade (FEAC) \\ Universidade Federal de Alagoas (UFAL), \\ Maceió, AL, Brasil.
}

\begin{abstract}
RESUMO - Este trabalho tem como objetivo fazer uma revisão teórica dos Sistemas de Inovação buscando averiguar qual a relação existente entre o Sistema Setorial de Inovação (SSI) com Complexo Econômico e Industrial da Saúde (CEIS), porém começando do Sistema de Inovação, movendo-se para suas descrições dos conceitos de Sistema Nacional de Inovação (SNI) e Sistema Regional de Inovação (SRI) e construir a metodologia do CEIS. A metodologia utilizada no trabalho foi realizada por meio da análise de vários artigos científicos e teses de autores nacionais e internacionais. O setor saúde vem sendo estudado com maior veemência por alguns autores, por meio de estudos do Sistema de Inovação, tornando-se um complexo no qual os agentes passam a interagir com o intuito de desenvolver e difundir o conhecimento, denominado CEIS.
\end{abstract}

Palavras-chaves: Sistemas de Inovação, Sistema Setorial de Inovação, Complexo Econômico e Industrial da Saúde.

\section{INNOVATION SYSTEMS AND THE COMPLEX ECONOMIC AND INDUSTRIAL HEALTH: THEORETICAL ASPECTS.}

\begin{abstract}
This work aims to make a theoretical revision of the Innovation Systems in order to investigate the relationship between the Sectorial Innovation System (SSI) with The Health Economic-Industrial Complex (HEIC), but starting from the Innovation System, moving to its descriptions of the concepts of National Innovation System (SNI) and Regional Innovation System (SRI) and build the methodology of the HEIC. The methodology used in the work was carried out through the analysis of several scientific articles and theses of national and international authors. The health sector has been studied with greater vehemence by some authors, through studies of the Innovation System, becoming a complex in which the agents begin to interact with the intention of developing and diffusing knowledge, called HEIC.
\end{abstract}

Keywords: Innovation Systems, Innovation Sector System, Health Economic-Industrial Complex.

\section{INTRODUÇÃO}

Atualmente o setor saúde é tido como fator estruturante do Estado de Bem-Estar, indutor do crescimento da economia, visto que é um dos campos mais dinâmicos do desenvolvimento econômico, além de ser um importante fator de geração de inovação (tecnologias estratégicas e 
P\&D). A saúde tem sido um elemento estruturante para o desenvolvimento regional atuando de maneira progressista na geopolítica internacional (GADELHA; COSTA, 2010).

É nítido que os avanços da tecnologia no final do século XIX contribuíram de forma positiva para a melhoria da qualidade de vida da população. Todavia, em meados do século $\mathrm{XX}$, apesar dos cuidados a saúde terem uma maior contemplação por meio de vacinas e antibióticos, deve-se evidenciar que havia dificuldades de acesso a tecnologias seguras e eficientes por insuficiência de renda.

Observa-se que atualmente com a globalização os progressos tecnológicos têm impactado positivamente para o crescimento da economia, além do que o padrão de competição entre países e firmas está relacionado às inovações tecnológicas, estando o setor saúde de certa maneira relacionado a isso, já que é um dos setores que tem movimentado um volume significativo de recursos na economia.

Diante disso, a saúde tem se tornado uma área de importância relevante para os estudos de economia. O setor saúde vem sendo estudado com maior veemência por alguns autores, por meio de estudos do Sistema de Inovação, tornando-se um complexo no qual os agentes passam a interagir com o intuito de desenvolver e difundir o conhecimento, denominado Complexo Econômico e Industrial da Saúde.

A dinâmica da saúde está intensamente relacionada à inovação, assim como outros setores da economia. A inovação no setor saúde apresenta características específicas, como por exemplo, a intensidade em pesquisa e desenvolvimento e a variedade de padrões tecnológicos e a intervenção de instituições que promovam a regulação. Essas características possibilitam que o setor saúde possa ser configurado como um sistema setorial de inovação ou um Complexo Econômico e Industrial da Saúde (CEIS), no qual há interação entre diferentes atores, como empresas, universidades e centro de pesquisa, hospitais e outros serviços de saúde, agências reguladoras e outras instituições governamentais.

Este trabalho tem como objetivo fazer uma revisão teórica dos Sistemas de Inovação buscando averiguar qual a relação existente entre o Sistema Setorial de Inovação com CEIS, porém começando do Sistema de Inovação, movendo-se para suas descrições dos conceitos de Sistema Nacional de Inovação e Sistema Regional de Inovação e construir a metodologia do CEIS.

Para a elaboração deste trabalho, foram analisados vários artigos científicos e teses de autores nacionais e internacionais, que discorrem sobre Sistema de Inovação, Sistema Nacional de Inovação, Sistemas Setoriais de Inovação, Sistema Nacional de Inovação em Saúde e Complexo Econômico e Industrial da Saúde. 
O trabalho está estruturado em quatro etapas além desta introdução. Primeiramente será realizada uma breve descrição sobre os conceitos de Sistema de Inovação, Sistema Nacional de Inovação, Sistema Regional de Inovação e Sistema Setorial de Inovação, evidenciando o último. Em seguida é realizada uma discussão sobre o Sistema Nacional de Inovação em Saúde, Sistema Setorial de Inovação em Saúde e o CEIS. Depois se discorre sobre a metodologia do Complexo Econômico e Industrial da Saúde. E por fim, é apresentada a conclusão do trabalho.

\section{SISTEMAS DE INOVAÇÃO}

\section{Sistema Nacional de Inovação, Regional e Setorial de Inovação}

Inicialmente, deve-se definir Sistema de Inovação (SI). O Sistema de Inovação, segundo Sbica e Pelaez (2006), é caracterizado por ser um conjunto de agentes públicos e privados onde contribuem, nos campos tanto macroeconômicos quanto microeconômico, para o desenvolvimento e a difusão de tecnologias inovadoras.

O Sistema de Inovação pode ser entendido através das interações entre agentes econômicos e institucionais, que instigam o desenvolvimento e a difusão de novas tecnologias, buscando atingir o esforço inovativo de dada região. Essas interações estão na base do SI e o processo de inovação acontece por meio de um esforço interativo, concedendo características sistêmicas (APOLINÁRIO; SILVA, 2013).

Para Cassiolato e Lastres (2005) o "sistema de inovação" é definido como um conjunto de instituições distintas que colaboram para o desenvolvimento da capacidade de inovação e aprendizado de um país, região, setor ou localidade. Sendo composto por elementos e relações que exercem interações na produção, difusão e uso do conhecimento. A lógica da definição de sistemas de inovação é que o desempenho inovativo é influenciado tanto pelo desempenho de empresas e organizações de ensino e pesquisa, como também pela forma como elas interagem entre si e com diversos atores e instituições, atingindo o desenvolvimento dos sistemas.

Deve-se evidenciar que tanto as organizações como as instituições são os elementos incentivadores dos sistemas de inovações como também as relações que há entre elas. Essas relações fazem parte dos sistemas de inovações e também da coordenação entre as várias partes existentes no sistema.

Ao analisar o SI a partir de uma abordagem geográfica integra-se o conceito de Sistema Nacional de Inovação (SNI). O SNI é delimitado por fronteiras nacionais e incide sobre o papel das organizações e instituições. 
Nos anos de 1980 e 1990, a definição de Sistemas Nacionais de Inovação se desenvolveu nos Estados Unidos e na Europa, para entender o processo de interação entre os diversos atores que estão relacionados ao surgimento de novos produtos e processos no mercado, principalmente os que abrangem conhecimento científico e tecnologia avançada. A base para o desenvolvimento da definição se deu a partir de análise das economias desenvolvidas, estendendo-se para países de industrialização mais contemporânea (GUIMARÃES, 2006).

Alguns autores como Lundval (1992), Freeman (1989; 1995) e Nelson (1993) depositaram em seus trabalhos algumas contribuições a respeito da formulação da definição de Sistemas Nacionais de Inovação.

O Sistema Nacional de Inovação está voltado para a função da aprendizagem interativa como das instituições nacionais que permitem que sejam explicadas as diferentes performances ligadas ao surgimento de inovações e do crescimento econômico dos países.

Para Freeman (1989; 1995), o Sistema Nacional de Inovação seria como um conjunto de instituições dos setores públicos e privados onde suas atividades e as articulações entre si geram alterações, importam como também promovem a difusão tecnológica.

Já na visão de Lundvall (1992), o Sistema Nacional de Inovação é caracterizado de maneira mais ampla, onde inclui todas as formas da estrutura econômica como do arcabouço institucional que interfiram no processo de aprendizado, assim, o SNI seria um agrupamento de instituições onde as relações entre elas possam vir a determinar a habilidade do surgimento de inovações das empresas nacionais.

Conforme Albuquerque (1996, p. 57):

Sistema nacional de inovação é uma construção institucional, produto de uma ação planejada e consciente ou de um somatório de decisões não-planejadas e desarticuladas, que impulsiona o progresso tecnológico em economias capitalistas complexas. Através da construção desse sistema de inovação viabiliza-se a realização de fluxos de informação necessária ao processo de inovação tecnológica.

Os Sistemas Regionais de Inovação (SRI) fazem parte do SNI. Estes sistemas de inovação têm por limite a região, são em escala reduzida dando importância aos aspectos de cada região onde se instalam. A definição de SRN é relevante para o entendimento da significância dos agentes locais no processo inovativo.

Para se ter uma maior clareza sobre a inovação a definição de sistema setorial de inovação vem para complementar o SNI e o SRI. Isto é, o conceito de sistemas nacionais de inovação foi estendido para a conceituação de sistemas setoriais de inovação, cujas características se traduzem na ausência de homogeneidade no processo de inovação entre os diferentes setores e seus subsetores. 
O SSI caracteriza-se com uma maior magnitude na compreensão de mudanças tecnológicas como também na estrutura da dinâmica inovadora, observando as diferenças existentes entre os setores em suas formas de aprendizado da inovação, indo além das fronteiras geográficas, o que acabava por limitar os sistemas no âmbito nacional e regional (CAVALCANTE, 2013).

A abordagem teórica e analítica dos sistemas setoriais é baseada na Teoria Evolucionária. Essa teoria dá ênfase na dinâmica para os processos de inovação e transformação econômica, em que a aprendizagem e o conhecimento são elementos importantes para a mudança do sistema econômico, implicando na heterogeneidade, experiência e organização dos agentes (MALERBA, 2005).

O ritmo da atividade inovadora é influenciado por algumas características invariantes do aprendizado e do acúmulo de conhecimento, distinto em todos os setores. As especificidades da atividade inovadora resultam dos distintos regimes tecnológicos que são subjacentes a característica da tecnologia e do conhecimento (MALERBA; ORSENINGO, 1997).

As diferenças entre os setores também têm sido introduzidas por autores como Nelson e Winter, em que essas diferenças têm sido relacionadas a regimes tecnológicos, especificamente ao ambiente de aprendizado e conhecimento em que as empresas atuam.

Cabe então destacar que são as próprias características das inovações que fazem com que os setores se diferenciem, seja tanto pela intensidade em P\&D com também pela viabilidade de suas estratégias.

Pavitt (1984) busca explicar as semelhanças e diferenças existentes entre os padrões setoriais de mudança tecnológica e seus impactos na inovação, bem como a geração de inovação de empresas em diversos setores. Além de classificar a estrutura setorial em três formas: setor da produção da inovação, associado às fontes setoriais de tecnologia que são utilizadas em um setor; setor de uso da inovação são as fontes institucionais e da natureza da tecnologia que são produzidas no setor; e o setor da atividade principal da empresa é inovar, esse ponto são características dasempresas inovadoras.

Também foram definidas por Pavitt (1984) três taxonomias, sendo elas: empresas dominadas pelo fornecedor, em que as empresas desses setores possuem uma proporção alta de inovações produzidas por outras empresas de outros setores, tendo sua trajetória tecnológica definida em termos de corte de despesas; produção intensiva, as empresas se originaram a partir da crescente divisão dotrabalho e abusca constantepelasimplificação das tarefas na área de produção; e base-científica essas empresas são encontradas na indústria química e eletrônica como também no setor elétrico, em ambos, as atividades de P\&D são as 
principais fontes de tecnologia. Isto é, cada uma das taxonomias tem um padrão estratégico que é dominado por cada setor.

Breschi, Malerba e Orsenigo (2000) analisaram a inovação e a evolução da indústria no âmbito de sistemas setoriais de inovação, onde tem sido alegado que a inovação em indústrias é o resultado da interação de diferentes atores que apresentam relações de colaboração de tipos formais e informais e têm ações fortemente influenciadas pelas competências das empresas e por processos de aprendizagem e pela base de conhecimento específica das indústrias. Para estes autores, o sistema setorial de inovação seria o conjunto de novos produtos estabelecidos para utilização específica, e o grupo de agentes, apresentando relações mercadológicas e nãomercadológicas para a geração, produção e vendas dos produtos.

A aprendizagem tecnológica por atores e o processo competitivo eliminará a heterogeneidade das empresas caracterizando um conjunto de modelos que visam explicar regularidades empíricas (MALERBA, 2007).

Assim, em um sistema setorial a inovação é visto como um processo que envolve interações sistemáticas entre inúmeros atores para a geração e troca de conhecimentos importantes para a inovação.

Breschi, Malerba e Orsenigo (2000) propõem que o padrão específico de atividades inovadoras em uma indústria pode ser explicado como resultado de diferentes regimes tecnológicos ou regime de aprendizagem. Além de dar um enfoque nas relações entre os padrões das atividades inovadoras dentro de um setor, mostrando que há características constantes de aprendizagem e acumulação de conhecimento que influenciam o andamento e a estrutura da atividade inovadora, divergindo em cada setor.

Os aspectos dos padrões de atividades inovadoras dos setores destacam as características de certos ambientes tecnológicos que são comuns aos grupos de indústrias e também alguns procedimentos constantes ao ambiente institucional.

Dentro da abordagem de Sistemas Setoriais de Inovação observa-se que o conceito de regimes tecnológicos possibilita explicar as transformações industriais por meio das dimensões concorrências do processo inovativo (MALERBA, 2005).

O regime tecnológico é definido como uma combinação de propriedades particulares fundamentais da tecnologia, que são: condição de oportunidade, condições de apropriabilidade, condições de cumulatividade e base de conhecimento (BRESCHI; MALERBA; ORSENIGO, 2000).

Segundo Malerba $(2003,2002)$ ao analisar o SSI observar-se algumas características tais como: conhecimento e domínio tecnológico, atores e redes e instituições. 
O conhecimento e o domínio tecnológico dizem respeito à base de conhecimento e a capacidade de se acumular tecnologia, que são características que geram limitações setoriais, já que as atividades inovadoras possuem distinções em cada setor, afetando também o desempenho dos envolvidos.

Os atores e redes são os responsáveis pelos vários comportamentos existentes dentro de cada setor, onde os setores possuem características de organizações homogêneas, que podem ser ditas como sendo subunidades das organizações de maior porte, vários agentes interagem por meio de cooperação, comunicação, como também através de trocas ou de comportamentos competitivos.

As instituições, que podem vir a afetar o Sistema Setorial de Inovação, desta maneira é sempre relevante buscar meios que sejam coerentes em suas atitudes com relação a regras com as políticas setoriais para que elas não possam vir a afetarem as políticas públicas em inovação de determinado setor, as instituições são fortes aparatos para o desenvolvimento, as mesmas têm uma propriedade universal de todas as formas de organizações, onde as mesmas conduzem de certo modo as economias.

O SSI possui uma classificação que está associada aos elementos do regime tecnológico, onde os mesmos acabam por definir as distinções existentes entre os sistemas inovadores genéricos e os sistemas inovadores específicos, estando ligada a visão Schumpeter Mark I (estágio caracterizado por uma destruição criativa) e também a Schumpeter Mark II (caracterizado por uma acumulação criativa), essas duas visões estão associados ao regime tecnológico definindo a direção do desenvolvimento científico, fronteira e base do conhecimento.

O regime tecnológico afeta os padrões setoriais das atividades inovadoras. Se existe um elevado nível de oportunidade tecnológica a entrada tecnológica de novos inovadores é favorecida, além de restringir a concentração. Desta forma, o impacto das oportunidades tecnológicas sobre a taxa de concentração de atividades inovadoras pode depender das interações entre oportunidades, apropriabilidade e condições cumulatividade. É importante destacar que são essas condições que permitem que as empresas definam suas estratégias.

Com base no tipo e caracterização do regime tecnológico, o SSI pode ser classificado em cinco: o primeiro diz respeito aos setores onde os inovadores apresentam-se de maneira dispersa e sem fronteira espacial para o conhecimento específico, apresenta condições baixas de oportunidade, apropriabilidade e cumulatividade; já no segundo, os setores que possuem poucos inovadores concentrados e possuindo uma fronteira de crescimento globalizada, altas condições de oportunidade, cumulatividade e apropriabilidade, com a presença de uma tecnologia 
dominante; no terceiro, estão os setores que possuem poucos inovadores concentrados, existindo uma fronteira local de conhecimento, apresentando condições com características medianas; o quarto são os setores que possuem muitos inovadores concentrados em áreas especificas com fronteira local e global em conhecimento, sistema com altas capacidades tecnológicas; e por fim são os setores em que existem muitos inovadores, porém suas fronteiras de conhecimento são de caráter local (MALERBA; ORSENIGO, 1997).

Malerba (2005) propôs uma visão multidimensional, integrada e dinâmica de inovação nos setores, relacionada com o quadro de sistemas setoriais de inovação, que fornece uma metodologia para a análise e comparação dos setores. Primeiramente, define setor como um conjunto de atividades que são unificadas por alguns grupos de produtos ligados para uma determinada demanda ou emergentes e que compartilham alguns conhecimentos comuns. Em seguida, classifica a estrutura do sistema setorial em três dimensões principais dos setores: conhecimento e domínio tecnológico, atores e redes e instituições.

O conhecimento desempenha um papel central na inovação, sendo altamente peculiar ao nível da empresa, não se difunde de forma automática e livre entre as empresas, e deve ser absorvido pelas empresas através de suas capacidades diferenciais acumuladas ao longo do tempo, divergindo em todos os setores em termos de domínios. Além disso, o conhecimento pode apresentar diferentes graus de acessibilidade, pode ser mais ou menos cumulativo.

Os sistemas setoriais são compostos por atores heterogêneos que são organizações ou indivíduos. A heterogeneidade da empresa também é afetada pelas características da base de conhecimento, experiência e processos de aprendizagem específicos, e da exploração de complementaridades dinâmicas. Dentro dos sistemas setoriais, agentes heterogêneos são conectados de várias maneiras por meio de relações de mercado e não-mercado.

Em um sistema setorial, a demanda passa a ser vista de forma diferenciada, passando a ser composta de agentes heterogêneos que interagem de várias formas com os produtores, isto é, consumidores individuais, empresas e órgãos públicos, caracterizada por conhecimento, processos de aprendizagem e competências, e que são afetados por fatores sociais e institucionais.

Desta forma, num quadro de sistema setorial, a inovação é considerada como um processo que envolve interações sistemáticas entre diversos atores para a geração e intercâmbio de conhecimentos relevantes para a inovação e seu lançamento no mercado. Assim, um sistema setorial passa por processos de mudança por meio da co-evolução de seus vários elementos. Para Murmann (2013), as indústrias que apresentam altas tecnologias são mais suscetíveis aos processos co-evolutivos. 
As diferenças nas dimensões de conhecimento proporcionam diferenças de desempenho através da acessibilidade e da complexidade.

A dinâmica e o funcionamento de um SSI estão associados às relações entre os elementos dos building blocks. Malerba (2002) descreve estes como sendo à base de conhecimento e processos de aprendizado; tecnologias básicas insumos e demanda, com as relações determinantes e complementaridades dinâmicas; tipo e estrutura das interações entre firmas e organizações não-mercantis; instituições e; processos de geração de variedade e de seleção.

O foco na diversidade dos sistemas setoriais de inovação destaca diferentes medidas políticas para diferentes setores. A política tem que considerar a coexistência de dimensões geográficas distintas de sistemas setoriais, em que o desenvolvimento local, nacional, regional, influencia os níveis globais de articulações das capacidades tecnológicas. As necessidades da política estão intimamente relacionadas aos problemas enfrentados pelos diversos agentes que operam nos contextos setoriais e à especificidade setorial dos conhecimentos, das fronteiras, atores e redes (MALERBA, 2005).

No quadro 1 abaixo é mostrado algumas características do Sistema Setorial de Inovação e do Complexo Econômico e Industrial da Saúde.

Quadro 1 - Características do Sistema Setorial de Inovação e do Complexo Econômico e Industrial da Saúde

\begin{tabular}{|c|c|}
\hline SISTEMA SETORIAL DE INOVAÇÃO & $\begin{array}{c}\text { COMPLEXO ECONOMICO E } \\
\text { INDUSTRIAL DA SAÚDE }\end{array}$ \\
\hline $\begin{array}{c}\text { A definição de sistema setorial de inovação } \\
\text { vem para complementar o SNI e o SRI. }\end{array}$ & $\begin{array}{c}\text { Sistema produtivo que vem sendo articulado } \\
\text { pela saúde de maneira interdependente. }\end{array}$ \\
$\begin{array}{c}\text { Caracteriza-se na compreensão de mudanças } \\
\text { tecnológicas como também na estrutura da } \\
\text { dinâmica inovadora, observando as diferenças } \\
\text { existentes entre os setores }\end{array}$ & $\begin{array}{c}\text { Formado pelos subsistemas de base industrial } \\
\text { e de serviços, associados à mesma estrutura } \\
\text { institucional }\end{array}$ \\
$\begin{array}{c}\text { Possui algumas características tais como: } \\
\text { conhecimento e domínio tecnológico, atores e } \\
\text { redes e instituições. }\end{array}$ & $\begin{array}{c}\text { Abrange setores produtivos, acarretando novos } \\
\text { paradigmas tecnológicos. }\end{array}$ \\
\hline
\end{tabular}

Fonte: Elaborado pelos autores, a partir dos textos de Malerba (1997) e do Gadelha (2003; 2006; 2009). 


\title{
SISTEMA NACIONAL DE INOVAÇÃO EM SAÚDE, SISTEMA SETORIAL DE INOVAÇÃO EM SAÚDE E O COMPLEXO ECONÔMICO E INDUSTRIAL DA SAÚDE (CEIS)
}

A literatura tem avançado e tem contribuido para mostraralgumas diferenças entre os setores em inovação e difusão com base em diferentes dimensões. Algumas dessas diferenças partem das ideias de Schumpeter, principalmente focando em distinções na estrutura de mercado e na dinâmica industrial entre os setores.

Devido à relevância e a complexidade, a saúde surge como uma área estratégica para se programar as desagregações analíticas, políticas e operacionais da denominação de Sistemas Nacionais de Inovação, possibilitando o esclarecimento da característica sistêmica, nacional e política do desenvolvimento dos Estados nacionais focados na criação e difusão da inovação.

Para Gadelha et al. (2013, p. 255):

\begin{abstract}
A desagregação do conceito de Sistema Nacional de Inovação, a partir de seus componentes setoriais, também constitui um caminho amplamente trilhado no âmbito da abordagem neo-schumpeteriana (FREEMAN; SOETE, 1997; PAVITT, 1984, MALERBA; ORSENIGO, 1997). No contexto específico das abordagens sobre inovação em saúde, cabe destacar, inicialmente, o conceito de complexo médico-industrial proposto por Cordeiro (1980), que enfatiza as diversas formas de articulação envolvendo atividades de assistência médica, redes de formação profissional, segmentos da indústria farmacêutica e de equipamentos médicos. Gelijns e Rosemberg (1995) partem de uma concepção semelhante para analisar os fluxos de informação e os mecanismos de geração, difusão e uso de inovações no campo médico, resultantes das interações entre diferentes segmentos do sistema de inovação em saúde. Também se destaca a contribuição de autores como Albuquerque e Cassiolato (2000), Quental et al (2000), Gadelha (2002, 2003, 2006) e Albuquerque et al (2004), no sentido de compreender as características do Sistema Nacional de Inovação no setor de saúde.
\end{abstract}

O tratamento sistêmico dado a base produtiva e de inovação em saúde tem se aplicado na prática de política de desenvolvimento na área industrial e produtiva, da saúde e da ciência e tecnologia no Brasil. Desta forma, a saúde é entendida como um plano econômico correlativo, que caracteriza um sistema de inovação e um sistema produtivo, gerando conhecimentos, a presença de um eixo econômico setorial de intensa significância, o consumo de massas e a presença marcada do Estado na regulação e na realização das atividades e da inovação.

O elemento crítico e normativo substantivo que é eminente neste comportamento é a assimilação tanto das atividades industriais como dos serviços, que se associam ao CEIS sobre a ótica de mercado, do conhecimento e institucional possuindo dinâmica particular de produção e de inovação (GADELHA et al., 2013). 
Assim, um sistema nacional de inovação pode ser segmentado em fragmentos setoriais, na qual o setor saúde representa um elemento significante, já que está situado entre o sistema de inovação e o sistema de bem-estar social.

É importante evidenciar que devido à articulação entre o sistema setorial de inovação no setor saúde e o sistema de bem-estar social, a regulação das atividades do setor é influenciada, no sentido de ser voltada para os arranjos institucionais e o progresso tecnológico, atingindo profundamente o funcionamento da atividade econômica, industrial e social do campo da saúde (ALBUQUERQUE; CASSIOLATO, 2002).

Um sistema de saúde engloba a demanda social por bens e serviços de saúde e as entidades direcionadas para a criação de recursos humanos, financeiros, tecnológicos, produtivos e de infraestrutura e também o provimento de serviços de saúde. No âmbito do sistema de saúde o Estado passa a mediar às relações entres os diversos agentes, apoiando as organizações públicas e privadas, além de ser o responsável pela promoção da regulação nesse sistema (GADELHA; QUENTAL; FIALHO, 2003).

A figura 1 representa o âmbito do sistema nacional de inovação em saúde que está localizado na interseção entre o sistema de inovação e o sistema nacional de saúde. Deve-se destacar que no sistema de inovação em saúde o Estado atua como mediador da oferta e demanda de bens e serviços de saúde buscando alcançar resultados favoráveis no que diz respeito ao desenvolvimento industrial, de tecnologias no campo da saúde, além da promoção da saúde (GADELHA; QUENTAL; FIALHO, 2003).

Figura 1 - Âmbito do Sistema Nacional de Inovação em Saúde

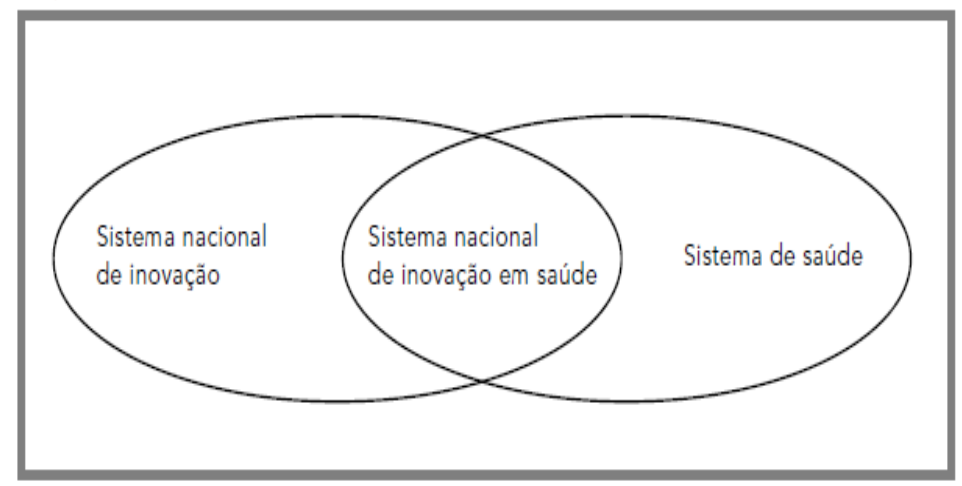

Fonte: Gadelha, Quental e Fialho, 2003.

Desta forma o Sistema Nacional de Inovação em Saúde desempenha a ligação entre o Sistema Nacional de Inovação e o Sistema de Saúde formando um elemento relevante do sistema de bem-estar. Vale mencionar que a dinâmica competitiva dos elementos produtivos da saúde e as ligações interdependentes existente levam a transformação dos paradigmas e trajetórias tecnológicas estratégicas para a inovação em saúde. 
"O sistema nacional de saúde, portanto, pode também gerar benefícios para a economia como um todo, especialmente em termos de pesquisa e desenvolvimento tecnológico, possibilitando, ainda, a geração de produtos ou serviços exportáveis [...]”. (BERCOVICI, 2013, p. 19).

O papel das universidades e instituições de pesquisa, a assistência médica (hospitais, clínica, postos médicos etc.), as instituições de regulação e associações profissionais e escolas médicas, as firmas do setor, a saúde pública e a efetividade das inovações realizada pela assistência médica e saúde pública, são algumas das características gerais do sistema de inovação do setor saúde (ALBUQUERQUE; CASSIOLATO, 2002).

A figura 2 mostra a caracterização do Sistema Nacional de Inovação em Saúde, apontando o arcabouço produtivo como também a camada sócio-institucional e política que o contém.

Figura 2 - Sistema Nacional de Inovação em Saúde: contexto político-institucional e produtivo

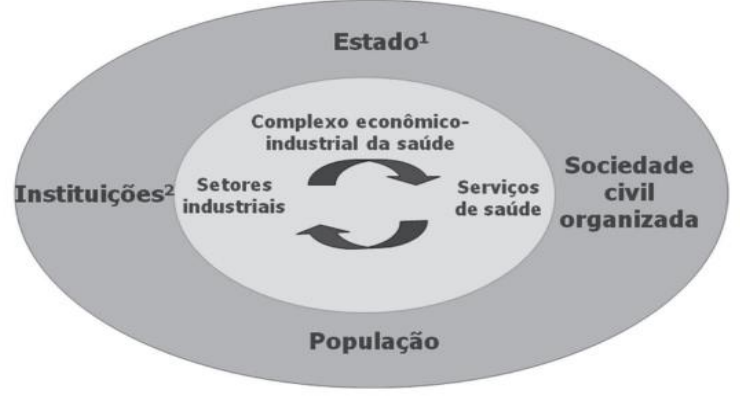

Fonte: Gadelha e Maldonado (2007).

1. Incorpora relações de poder, estrutura decisória e a formulação e implementação de políticas implícitas e explícitas.

2. Desde instituições formais de C\&T e de educação, agências de fomento, órgãos de financiamento, entre outras, até de normas de conduta institucionalizadas na sociedade.

É importante destacar que a partir da desagregação do Sistema Nacional de Inovação pode ser constituído o Sistema Setorial de Inovação em Saúde (SSIS). Este sistema apresenta dinâmica singular, no qual diversos agentes interagem com objetivo de produzir conhecimento e inovação para o campo da saúde (ALBUQUERQUE; CASSIOLATO, 2002). Dentro das abordagens do SSIS, a do CEIS, é a mais utilizada atualmente.

A definição do Complexo Econômico e Industrial da Saúde foi desenvolvida em volta dos Sistemas Nacionais de Inovação em Saúde favorecendo a ligação entre o arcabouço produtivo e as inovações, compreendendo os vínculos de interdependência entre os setores de atividades, sendo segmento marcado dos Sistemas Nacionais de Inovação. Por meio do CEIS pode-se assinalar um grupo específico de subsistemas econômicos, que estão no âmbito particular característico do campo da saúde (GADELHA et al., 2013). 


\section{COMPLEXO ECONÔMICO E INDUSTRIAL DA SAÚDE}

O Complexo Econômico e Industrial da Saúde, conceituação estabelecida para o Sistema Produtivo Nacional de Saúde, formou-se em uma área bastante dinâmica, abrangendo atividades de profundo grau de inovação dentro dos novos paradigmas tecnológicos, por haver um eixo produtivo de bens e serviços altamente significante, correspondendo a uma parte relevante do Produto Interno Bruto (PIB) nas economias que estão emergindo e nas desenvolvidas, como também por relacionar a dimensão econômica e social, em conjunto com a ambiental, determinando o processo de desenvolvimento (GADELHA et al., 2013).

O CEIS integra o sistema político-institucional chamado de Sistema Nacional de Inovação em Saúde. Desta forma, o CEIS sofre influência do nível de desenvolvimento e de interação entre os vários agentes e políticas que compõem o arcabouço do sistema nacional de inovação em saúde, sendo esses interdependentes.

É estabelecido pelo CEIS um grupo de setores que reproduzem atividades na área da saúde mantendo ligações intersetoriais para o desempenho de atividades de pesquisa e desenvolvimento, produção, compra e venda de insumos de saúde, de serviços, além de conhecimentos e tecnologia, considerando a carência da interação das lógicas sanitária, econômica e inovativa do campo da saúde.

De acordo com Gadelha, Costa e Maldonado (2012) o CEIS é um sistema produtivo que vem sendo articulado pela saúde de maneira interdependente, sendo formado pelos subsistemas de base industrial e de serviços, em que essas atividades juntas correspondem a uma das áreas de maior dinamismo econômico.

O CEIS abrange setores produtivos que acarretam como consequências novos paradigmas tecnológicos, possuindo, assim, significativa potencialidade de criação de inovação que é peça fundamental para a competitividade, no que se refere a conhecimento, especialmente em cenário global. Entretanto ainda existe uma fragilidade na política de saúde, uma vez que o CEIS apresenta ainda uma base tecnológica bastante frágil, apesar de suas características estratégicas para o desenvolvimento nacional.

Observando-se a inovação do ponto de vista político e social, é reafirmada a necessidade de utilizar a ferramenta analítica usada pela economia política da saúde, a saúde é considerada um campo de estudo notável, possuindo uma relação existente entre o Estado e o setor privado, como também a sua inserção no cenário internacional. A inovação do setor de saúde voltada para o desenvolvimento econômico e social, tem ganhado reconhecimento a partir da priorização do complexo produtivo nas principais políticas e ações do Governo Federal (GADELHA, 2010). 
Assim para Gadelha, (2010, p. 3) tem-se que:

[...] No campo da política social de saúde, vale mencionar, ademais, a valorização da base produtiva, entendendo-a como uma condição sine qua non à sustentabilidade do sistema de saúde, a exemplo da priorização do Complexo no "Mais Saúde" e mais recentemente ao aumento do volume de recursos e de qualificação da atuação por parte do governo federal no que tange ao desenvolvimento desta base produtiva.

Para Gadelha (2006) o CEIS privilegia a atividade produtiva, levando em conta que a fragilidade do sistema industrial e empresarial do Brasil é a vulnerabilidade econômica presente, no país, quando se trata em área da saúde.

O CEIS procura destacar a relação existente entre inovações, as estruturas industriais e serviços de saúde que se associam ao mesmo ambiente político-institucional, em que a interdependência desse complexo produtivo no processo evolutivo dos paradigmas e trajetórias tecnológicas direciona as suas características sistemáticas.

No CEIS o Estado é responsável por promover o desenvolvimento tendo o dever de viabilizar a adequação das orientações macropolíticas e de estratégias diversificadas que estimulem o desenvolvimento, possibilitando o fortalecimento do arcabouço regulatório, utilização do poder de compra, estímulo à pesquisa $\mathrm{C} \& \mathrm{~T}$ e a distribuição regional dos recursos no território (GADELHA; COSTA; MALDONADO, 2012). Nesse sentido, deve-se destacar que o CEIS sofre por meio do Estado promoção e regulação.

A figura 3 mostra a morfologia do CEIS. O CEIS compreende os subsistemas de base industrial (subsistema de base química e biotecnológica e o subsistema de base mecânica, eletrônica e de materiais) e de serviços, na qual essas atividades juntas equivalem a uma das áreas de maior dinamismo da economia.

Figura 3 - Caracterização geral do Complexo Econômico-Industrial da Saúde

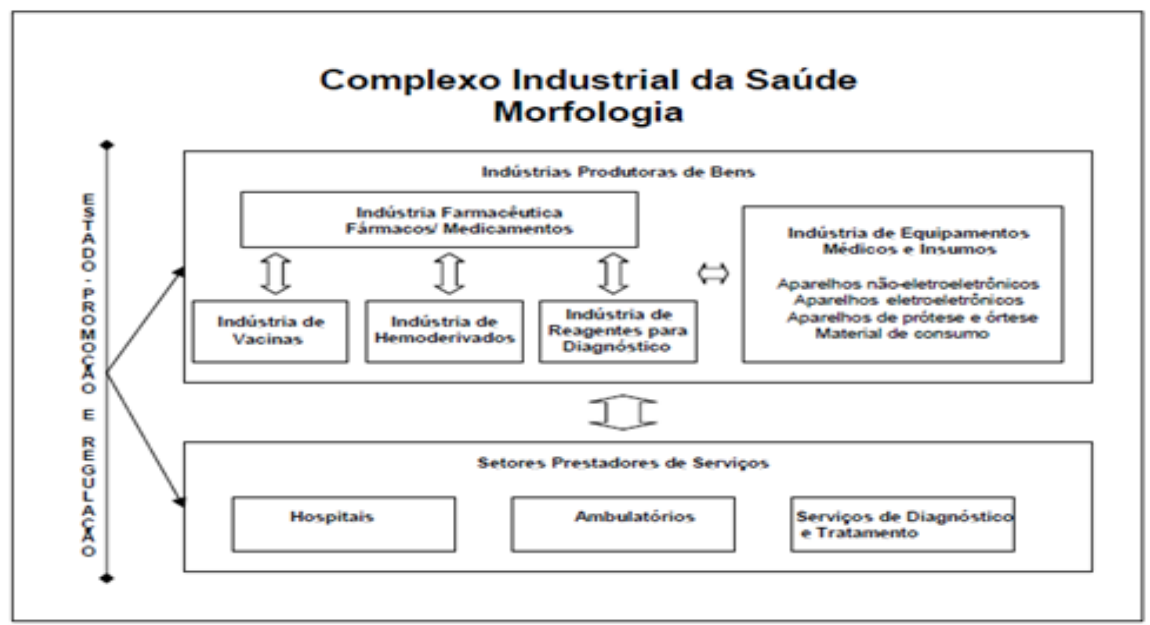

Fonte: GADELHA, 2003, p. 524. 


\section{SUBSISTEMA DE BASE QUÍMICA E BIOTECNOLÓGICA}

Conforme Gadelha, Costa e Maldonado (2012) o subsistema de base química e biotecnológica é encarregado na fabricação de vacinas, de soros, de fármacos, de medicamentos, de reagentes para diagnóstico e de hemoderivados, sendo que seus serviços acabam por ter uma grande importância para a sociedade, já que a medida que o setor se desenvolve melhor será o serviço de saúde prestado (GADELHA; COSTA; MALDONADO, 2012).

No subsistema de base química é possível observar que a sua dinâmica é caracterizada por um auto grau de universalização de sua produção como também o mercado apresenta um auto grau de concentração, uma vez que as empresas do setor acabam por produzir bens de características particulares, não ocorrendo no mercado uma competição via diferenciação de preços.

A indústria farmacêutica internacional é composta por oligopólios com altas barreiras à entrada. As barreiras à entrada mais expressivas são a economia de escala, o desenvolvimento dos setores de $\mathrm{P} \& \mathrm{D}$, competição mediante preço, e principalmente a patente de medicamentos desenvolvidos, o que impede a entrada de concorrentes em mercados específicos (GADELHA, 2009).

No caso brasileiro é estabelecido o poder dos oligopólios internacionais da indústria farmacêutica. A produção interna é identificada especialmente pela produção de genéricos e, no campo de pesquisa, estão em evidência a Fundação Oswaldo Cruz (FIOCRUZ) e o Instituto Butantã (GADELHA, 2009).

\section{SUBSISTEMA DE BASE “MECÂNICA, ELETRÔNICA E DE MATERIAIS"}

Ao analisar a indústria de base mecânica, eletrônica e de materiais associada a parte da saúde é importante analisar o seu nível crescente de tecnologia, como também observar o lado social ligado aos serviços sociais de saúde prestados, o subsistema de base mecânica, eletrônica e de materiais acaba por evidenciar a relação existente entre os fundamentos econômicos e sociais do desenvolvimento.

Este subsistema é caracterizado por ser heterogêneo, o mesmo possui equipamentos de usos cotidianos com baixo nível tecnológico até equipamentos com nível tecnológicos altos, de grande complexidade. A parte do subsistema que possui um alto grau de complexidade são baseadas em tecnologias estratégicas. Esse subsistema é considerado como oligopólio onde o mesmo é fundamentado na distinção do produto em que os bens do setor são tidos com um alto grau de especialização e que sempre estão surgindo novas opções tecnológicas.

A indústria de base mecânica, eletrônica e de materiais acaba por se destacar pelo elevado nível de sua base tecnológica como também pelo caráter social próprio aos serviços 
de saúde, esse subsistema abrange uma pluralidade de segmentos com características e dinâmicas diferentes entre si. A presente indústria vem se beneficiando das inovações tecnológicas geradas em outros setores, como, por exemplo, mecânica de precisão, microeletrônica, entre outras (GUTIERREZ; ALEXANDRE, 2004).

De acordo com Maldonado et al., (2012, p. 81):

\begin{abstract}
Esta indústria é fortemente associada às práticas médicas, determinando muitas vezes a tecnologia incorporada nos procedimentos adotados, no que se refere à prevenção, diagnóstico e tratamento de doenças. Possui, ademais, grande heterogeneidade tecnológica, incorporando segmentos bastante diversificados, desde bens de capital de alta complexidade (como diagnóstico por imagem) até materiais de consumo de uso rotineiro, passando por instrumentos, material cirúrgico e ambulatorial, seringas, entre muitos outros exemplos.
\end{abstract}

No que diz respeito à estrutura de mercado, observa-se uma grande segmentação, em que predominam empresas de pequeno e médio porte com atividades especializadas, e as empresas de grande porte apresentam uma presença consideravelmente alta principalmente nos segmentos de produtos que possuem uma maior complexidade e elevado valor unitário.

Cabe destacar que o subsistema de base mecânica, eletrônica e de materiais dentre os subsistemas pertencentes ao Complexo Econômico Industrial da Saúde (CEIS) vem se destacando, pois o mesmo apresenta saldos favoráveis no que diz respeito ao seu faturamento na década de 2000, como também no que se refere a um aumento das suas exportações, ao surgimento de novas empresas e criação de empregos (GADELHA; COSTA; MALDONADO, 2012).

\title{
SUBSISTEMA DE SERVIÇOS
}

O subsistema de serviços pertencente ao Complexo Econômico Industrial da Saúde (CEIS) possui um peso econômico maior que os outros dois subsistemas. Esse tem influência na geração de emprego e renda da sociedade, além de possuir um papel determinante no caráter sistêmico do CEIS.

De acordo com Costa et al. (2013) as características sistêmicas do CEIS são constituídas pelo subsistema de serviços em saúde, que realiza atividade específica na dinamização da ligação entre os vários subsistemas exercendo função de consumidor e demandante, no qual apresenta influência na produção de equipamentos médico-hospitalares, produtos farmacêuticos, imunoderivados, soros e demais insumos.

Desta forma, peculiaridades na orientação dos serviços no setor saúde e no arcabouço produtivo do CEIS são adequadas nos participantes dos Sistemas Nacionais de Saúde, mostrando a significância da adequação da estrutura institucional e da abordagem sistemática dos setores que compõem o complexo da saúde. 
Os trabalhos que as instituições de pesquisa têm elaborado e os conhecimentos desenvolvidos para o desenvolvimento do complexo produtivo da saúde, especialmente os que envolvem elementos de caráter biotecnológico (medicamentos, vacinas e dispositivos diagnósticos) tem sido de relevância significante. No entanto, se faz necessário articular o Sistema Setorial de Inovação em Saúde como condição econômica capaz de gerar emprego e renda pela produção, ao setor de saúde, na qualidade de categoria de inclusão social gerando emprego e renda devido à expansão do bem-estar (GUIMARÃES, 2006).

\section{CONCLUSÃO}

A literatura do trabalho possui certas restrições, já que a mesma se encontra de maneira bastante seleta, onde poucos autores trabalham sobre o tema proposto, além da dificuldade de abordar de maneira precisa o problema central do trabalho, em se tratando do Sistema Setorial de Inovação e o Complexo Econômico e Industrial da Saúde.

No decorrer do trabalho, Gadelha (2009) adota o conceito de Complexo Econômico e Industrial da Saúde como sendo um sistema produtivo setorial, de grande relevância às bases produtivas nacionais.

O setor saúde vem sendo estudado com maior intensidade, por alguns autores através de estudo do Sistema de Inovação, transformando-o em um complexo no qual os agentes passam a interagir com o propósito de desenvolvimento e difusão do conhecimento, caracterizado como o CEIS.

Observa-se que o Sistema Nacional de Inovação pode ser desagregado em diversos Sistemas Setoriais de Inovação, no qual se pode constituir o Sistema Setorial de Inovação em Saúde. Dentro das abordagens do Sistema Setorial de Inovação em Saúde a abordagem do CEIS, é a mais utilizada atualmente. É importante destacar que o CEIS é um setor de grande dinâmica econômica, envolvendo elevada densidade de inovação e possuindo uma base produtiva relevante.

Os subsistemasde base industrial (subsistema de base química e biotecnológica e o subsistema de base mecânica, eletrônica e de materiais) e de serviços, que compõem o CEIS, interagem para produzir e inovar na assistência à saúde. A população demandante entra no complexo pelo subsistema de serviços movimentando a produção de todo o CEIS.

Sabe-se que o principal desafio para reverter à vulnerabilidade existente no CEIS fundamenta-se em intensificar e qualificar a atuação do Estado. O CEIS, por ser um sistema e ter subsistemas, possui diferente marcos regulatório.

Atualmente o desafio principal é enraizar o estabelecimento das características estratégicas do Complexo Econômico e Industrial da Saúde e estimular o desenvolvimento levando-se em conta as variantes inclusas no programa de inovação em saúde de maneira interdependente. 
Deve-se destacar que o conhecimento produzido a partir do Sistema de Inovação relaciona-se com o setor de produção da saúde, estabelecido como o CEIS. Assim, o CEIS apresenta um forte dinamismo econômico, envolvendo intensa densidade de inovação, possuindo um eixo produtivo relevante.

O Sistema Setorial de Inovação possui grande destaque na percepção de mudanças tecnológicas como também no arcabouço da dinâmica inovadora. O SSI observa as diferenças presentes entre os setores em suas formas de aprendizado da inovação.

Associado ao conceito de Sistema Setorial de Inovação é observa-se uma visão multidimensional, apresentada por Malerba (2003), que trata da dinâmica e da integração de uma rede de interações entre vários setores.

Portanto, é de suma importância que os setores acumulem competências tecnológicas, de modo que ocorram ganhos dinâmicos de competitividade oriundos da interação entre inovação e ganhos sistemáticos, mediante a dinâmica setorial do sistema de inovação em saúde.

\section{REFERÊNCIAS BIBLIOGRÁFICAS}

ALBUQUERQUE, E. M. Sistema nacional de inovação no Brasil: uma análise introdutória a partir de dados disponíveis sobre a ciência e a tecnologia. Revista de Economia Política, v. 16, n. 3 (63), julset/1996. Disponível em: 〈http://www.rep.org.br/pdf/63-4.pdf>. Acesso em: 12 fev. 2016.

ALBUQUERQUE, E. M.; CASSIOLATO, J. E. As Especificidades do Sistema de Inovação do Setor Saúde. Revista de Economia Política, v. 22, n. 4 (88), out-dez/2002. Disponível em: <http://www.rep.org.br/PDF/88-9.PDF>. Acesso em: 15 fev. 2016.

APOLINÁRIO, V.; SILVA, M. L. Sistema de inovação e desenvolvimento: reflexões a partir da experiência brasileira, 2013. Disponível em: <http://www.redesist.ie.ufrj.br/lalics/papers/125_Sistema_de_inovacao_e_desenvolvimentoreflexoes_a_p artir_da_experiencia_brasileira.pdf $>$. Acesso em: 29fev. 2016.

BERCOVICI, G. Complexo Industrial da Saúde, desenvolvimento e proteção constitucional ao mercado interno. R. Dir. Sanit., São Paulo, v. 14, n. 2, p. 9-42, jul./out. 2013.

BRESCHI S., MALERBA F., ORSENIGO L., Technological regimes and sectoral patterns of innovation, Economic Journal, 2000.

CASSIOLATO, J. E.; LASTRES, H. M. M. Sistemas de inovação e desenvolvimento as implicações de política. São Paulo em Perspectiva, v. 19, n. 1, p. 34-45, jan./mar. 2005. Disponível em: <http://www.scielo.br/pdf/spp/v19n1/v19n1a03.pdf>. Acesso em: 26 jan. 2016.

CAVALCANTE, J. B. Caracterização do perfil de inovação dos setores industriais do complexo econômico industrial da saúde: discussão da política tecnológica, inovação e comércio exterior. 2013 Dissertação (Mestrado em Economia Aplicada). Universidade Federal de Alagoas, Maceió, 2013. Disponível em: <http://www.feac.ufal.br/mestrado/economia/sites/default/files/dissertacoes/dissertacao_pdf.pdf>. Acesso em 10 jan. 2016. 
COSTA, L. S. Análise da dinâmica de geração de inovação em saúde: a perspectiva dos serviços e do território. 2013. Tese (Doutorado em Saúde Pública). Escola Nacional de Saúde Pública Sergio Arouca, Rio de Janeiro, 2013.

COSTA, L. S., et al. Análise do subsistema de serviços em saúde na dinâmica do complexo econômicoindustrial da saúde. In FUNDAÇÃO OSWALDO CRUZ. A saúde no Brasil em 2030 - prospecção estratégica do sistema de saúde brasileiro: desenvolvimento produtivo e complexo da saúde [online]. Rio de Janeiro: Fiocruz/Ipea/Ministério da Saúde/Secretaria de Assuntos Estratégicos da Presidência da República, 2013. Vol. 5. p. 121-159. ISBN 978-85-8110-019-7. Disponível em: < http://books.scielo.org/id/scfy6/pdf/noronha-9788581100197-06.pdf>. Acesso em: 20 jan. 2016.

FREEMAN, C. The "National System of Innovation" in historical perspective. Cambridge Journal of Economics19 (1): $\quad$ 5-24, $1995 . \quad$ Disponível <http://www.globelicsacademy.org/2011_pdf/Freeman\%20NSI\%20historial\%20perspective.pdf>.

Acessoem: 12 jan. 2016.

FREEMAN, C. Technology policy and economic performance: lessons from Japan. London: Pinter Pub, 1989.

GADELHA, C. A. G. Desenvolvimento, complexo industrial da saúde e política industrial. Revista de Saúde Pública, 40(NEsp): 11-23, $2006 . \quad$ Disponível em: <http://www.scielo.br/pdf/rsp/v40nspe/30617.pdf>. Acesso em: 15 jan. 2016.

GADELHA, C. A. G.; QUENTAL, C.; FIALHO, B. C. Saúde e inovação: uma abordagem sistêmica das indústrias da saúde. Cad. Saúde Pública, Rio de Janeiro, 19(1): 47-59, jan-fev, 2003. Disponível em: <http://www.scielo.br/pdf/csp/v19n1/14904.pdf>. Acesso em: 1 fev. 2016.

GADELHA, C. A. G.; COSTA, L. S. Trajetória Brasil 2003-2009: A Saúde na Perspectiva da Proposta de Desenvolvimento do Governo Federal. Nota Técnica. Brasília: CGEE, 2010.

GADELHA, C. A. G. "O complexo econômico-industrial da saúde". Informe CEIS, Rio de Janeiro 1 (2010).

GADELHA, C. A. G. et al. O Complexo Econômico-Industrial da Saúde no Brasil: dinâmica de inovação e implicações para o Sistema Nacional de Inovação em saúde. Revista Brasileira de Inovação, Campinas (SP), 12 (2), p. 251-282, julho/dezembro 2013. Disponível em: <http://ocs.ige.unicamp.br/ojs/rbi/article/view/540/377>. Acesso em: 15 dez. 2015.

GADELHA, C. A. G.; COSTA, L. S.; MALDONADO, J. O Complexo Econômico Industrial da Saúde e a dimensão social e econômica do desenvolvimento. Revista Saúde Pública, 2012; 46(Supl):21-8. <http://www.scielo.br/pdf/rsp/v46s1/ao4188.pdf>. Acesso em: 13 dez. 2015.

GADELHA, C. (Coord.) Perspectivas do investimento em saúde. Rio de Janeiro: UFRJ, Instituto de Economia, 2008/2009. 217 p. Relatório integrante da pesquisa - Perspectivas do Investimento no Brasill, em parceria com o Instituto de Economia.

GUIMARÃES, R. Pesquisa em saúde no Brasil: contexto e desafios. Rev. Saúde Pública; 40 (NEsp):3-10, 2006. Disponível em: <http://www.scielosp.org/pdf/rsp/v40nspe/30616.pdf?>. Acesso em: 10 fev. 2016.

GUTIERREZ, R. M. V.; ALEXANDRE, P. V. M. Complexo industrial da saúde: uma introdução ao setor de insumos e equipamentos de uso médico. BNDES Setorial, Rio de Janeiro, v. 19, p. 119-155, 2004.Disponível em: <https://web.bndes.gov.br/bib/jspui/bitstream/1408/2490/1/BS\%2019\%20Complexo\%20industrial\%20da $\% 20$ sa\%C3\%BAde_P.pdf>Acesso em: 20 dez. 2015.

LUNDVALL, B. Introduction. In: LUNDVALL, B (Ed.). National innovation system: towards a theory of innovation and interactive learning. Londres: Pinter Publishers, 1992. 
MALDONADO et al. O Subsistema de Base Mecânica, Eletrônica e de Materiais do Complexo Econômico Industrial da Saúde: perspectivas para 2022 / 2030. In: FUNDAÇÃO OSWALDO CRUZ et al. A saúde no Brasil em 2030: diretrizes para a prospecção estratégica do sistema de saúde brasileiro. Rio de Janeiro: Fiocruz, $2012 . \quad$ Disponível em: $<$ https://saudeamanha.fiocruz.br/sites/saudeamanha.fiocruz.br/files/publicacoes/ASaudeNoBrasilEm2030 _V5_O_Subsistema_de_Base_Mecanica_Eletronica_e_de_Materiais_do_Complexo\%20Economico_Indu strial_da_Saude.pdf>Acesso em: 18 jan. 2016.

MALERBA, F. Innovation and the dynamics of industries: progress and challenges.International Journal of Industrial Organization, 2007.

MALERBA, F. Sectoral systems and innovation and technology.São Paulo.Revista Brasileira de Inovação, v. 2, n. 2, p. 329-375, jul./dez., 2003. ISSN 2178-2822.

MALERBA, F. Sectoral systems of innovation: how and why innovation differs across sectors. In Handbook of Innovation J. Fagerberg, D. Mowery and R. Nelson (ed). Cambridge University Press, Cambridge 2005.

MALERBA, F. Sectoral systems of innovation and production.Research Policy, 31(2), 247-264, fev/2002.

MALERBA, F.; ORSENIGO, L. Technological regimes and sectoral patterns of innovative activities.Industrial and Corporate Change, v. 6, p. 83-117, 1997.

MURMANN, P. The coevolution of industries and important features of their environments. Organizationl Science, 2013 n.1 58-78. Disponível em:<http://professor-murmann.net/publications/Murmann_2013_Coevolution.pdf $>$. Acesso em: 11 nov. 2015.

NELSON, R. R. National Innovations Systems. A comparative analysis. New York, Oxford: Oxford University Press, 1993.

NELSON, R., WINTER, S. An evolutionary theory of economic change, Belknap Press, Cambridge, 1982.

PAVITT, K. Sectoral patterns of technical change: Towards a taxonomy and a theory. Research Policy, n. 13, p. 343-373, 1984.

SBICA, A; PELAEZ, V. Sistemas de Inovação. In: PELAEZ, V; SZMRECSÁNYI, T. Economia da inovação tecnológica.São Paulo: HUCITEC, Ordem dos economistas do Brasil, 2006. 